\title{
Journalisme, fiction et terrorisme Les Chemins inutiles de Saïd Oussad et la tragédie algérienne
}

\author{
Mohammed Yefsah \\ Université Oran 2
}

Tirs d'armes automatiques, débris en éclats, ceintures explosives, nuages de poussière, gravats et objets volants dans le ciel, crissements d'acier, feux et tintamarres, du sang, des larmes ou un silence, de la cendre sur des lieux et des visages, des cadavres et des instants en ralentis qui défilent. Voici quelques images, le plus souvent sorties des studios de l'industrie cinématographique, qui incarnent le terrorisme ou la guerre. Elles jettent à la face du monde la terreur qu'engendre la violence. Le terrorisme de masse est aussi une sorte de scénario puisque les commanditaires réfléchissent aux plus grands dégâts qu'ils pourraient engendrer et à la psychose qu'ils souhaiteraient installer. Leurs scenarii de la terreur cherchent les plus spectaculaires des actions pour frapper les esprits. Signalons cependant le fait que les pays occidentaux ont produit plus de films et de romans sur le terrorisme que les pays les plus touchés et qui le vivent au quotidien, pendant des années ou des décennies.

L'Algérie a vécu le terrorisme à partir de 1993 dans la solitude et l'isolement. Cette tragédie s'est jouée à huit $\operatorname{clos}^{1}$. Qualifiée de « décennie noire » ou «décennie rouge » par les journaux, cette période a fait l'objet de récits et de films produits sur le vif. Le romancier qui s'est fait connaitre au cœur de cette tragédie, avec ses romans policiers, est Yasmina Khadra ${ }^{2}$. Sa qualité d'écriture et son choix d'un pseudonyme féminin ont suscité la curiosité et lui ont permis d'acquérir un lectorat large,

\footnotetext{
${ }_{1}^{1}$ Outre le doute installé sur les auteurs de la violence et des massacres, un embargo sur les armes a empêché les services de sécurité algérienne - police, gendarmerie et armée d'acquérir du matériel nécessaire pour lutter contre le terrorisme, à l'exemple des lunettes à vision nocturne. Le sentiment de faire face seul au terrorisme s'est accentué avec la difficulté pour le simple citoyen d'obtenir des chancelières étrangères un visa, alors que l'asile politique était accordé facilement aux militants islamistes et à leurs représentants en Allemagne, en Grande-Bretagne, en France, aux États-Unis d'Amérique, etc. C'est une période également où des intellectuels, des cadres et des personnes qui en avaient les moyens ont fui le pays.

${ }^{2}$ De son vrai nom Mohammed Moulessehoul, ancien officier de l'armée algérienne. Il a publié plusieurs romans traitant du terrorisme.
} 
notamment en Occident ${ }^{3}$. Le terrorisme fut donc source d'inspiration pour de nombreux auteurs algériens. Les Chemins inutiles, première œuvre de Saïd Oussad ${ }^{4}$, est l'un de ces récits, mais il présente la particularité d'être un roman tardif par rapport à cette thématique, puisqu'il est publié en 2014.

\section{Intrigue et factuel}

L'intrigue des Chemins Inutiles raconte à la première personne les péripéties d'un journaliste sans patronyme, contacté par un groupe terroriste souhaitant faire des révélations. C'est à travers l'œil de ce journaliste-narrateur que l'on plonge dans l'univers sombre de cette décennie, dans l'atmosphère même d'une époque, ce qui confère une dimension psychologisante à ce roman. Ce personnage principal est pris dans la spirale d'une violence à la fois indubitable et indicible. Cette fiction s'inspire d'une situation vécue par l'auteur: directeur de publication et grand-reporter au quotidien francophone La Voix de l'Oranie, Saïd Oussad a effectivement été contacté par l'Armée islamique du salut (AIS), branche militaire du Front islamique du salut (FIS), qui souhaitait dédouaner son groupe d'un massacre de villageois, finalement attribué au Groupe islamique armé (GIA). Il exige alors une interview avec l'un de ses chefs, en l'occurrence Ahmed Benaïcha ${ }^{5}$. Cette organisation était en négociations secrètes avec l'armée algérienne pour déposer les armes. Le fil conducteur de la trame du récit est le périple et les péripéties qui mènent ce journaliste jusqu'à Benaïcha, personnage éponyme du chef terroriste. Au cours de son itinéraire, il croise d'autres terroristes dont il dresse les portraits et retrace les actions, particulièrement Mustapha Abou Qotada. Quelle est la différence fondamentale entre l'écriture journalistique et l'écriture romanesque dans, sur un contexte de terreur?

\footnotetext{
${ }^{3}$ L'horizon d'attente politique a joué un rôle dans la réception de la littérature algérienne à cette époque. Voir à ce sujet la thèse de Tristan Leperlier intitulée Une guerre des langues? Le champ littéraire algérien pendant la "décennie noire 》(1988-2003): Crise politique et consécrations transnationales.

4 Après un baccalauréat en mathématiques et deux années d'un parcours scientifique à l'Université d'Oran, Saïd Oussad décide de changer de cap en choisissant d'étudier les Lettres françaises, qu'il enseigne plus tard, dans un lycée. Il entame une carrière de journaliste avec l'hebdomadaire Détective (1993-2003), « journal à scandales » qui bouscule le paysage médiatique algérien, et rejoint ensuite Le Quotidien d'Oran. Après avoir brigué des postes à responsabilité, en tant que directeur de publication ou rédacteur en chef dans divers médias francophones, il est actuellement grand reporter au quotidien Liberté.

${ }^{5}$ Ancien émir de la zone ouest et numéro deux de cette organisation. Bénéficiant des lois d'amnistie, il est toujours en vie et il lui arrive même de répondre à des sollicitations des médias, journaux et télévisions, pour des entretiens.
} 
Alors que le journalisme récuse, du moins sur le plan formel, l'expression de la subjectivité, la fiction permet de s'épancher. Dans un entretien, l'auteur estime qu'il avait pris suffisamment de recul pour aborder d'un point de vue intime cette période charnière de l'histoire algérienne vécue dans la douleur la plupart du temps, mais également comme une expérience fondatrice d'un

nouveau mental [...] Précisément, je m'en suis servi comme alibi pour me replonger à l'intérieur de mes angoisses. Un vecteur pour exorciser des événements que je pensais avoir complètement oubliés. Une peur cachée, innommable, qui vous ronge de l'intérieur tant que vous ne l'avez pas crachée. Exprimant ses désillusions et son pessimisme, il évoque son écriture en termes de thérapie et de nouveau départ dans la vie et non comme rétrospective. ("Je suis un écrivain d'instinct»)

\section{Du journalisme à la littérature}

L'écriture pour un journal où le rédacteur s'implique d'une manière ou d'une autre à titre personnel peut engendrer des frustrations, particulièrement dans un contexte de conflit; la forme journalistique impose ses règles et la subjectivité doit être masquée. Face à la dureté des images vues, des informations quotidiennes rapportées et à l'obligation d'évitement du pathos, la littérature s'avère salvatrice. Le sujet du terrorisme dans la littérature algérienne est devenu un élément constitutif de son histoire dans le champ littéraire. L'écriture est partie composante du dévoilement d'un traumatisme refoulé sur le plan politique et social. Elle continue de questionner le sensible dans des contextes de crise. La fiction s'inscrit dans la carence du travail de témoignage et d'analyse de cette étape historique du pays. La mémoire meurtrie s'exprime à travers les créateurs, cinéastes et écrivains, afin d'établir un fil entre le passé et le présent, et dans l'espoir qu'aucune tragédie ne pointe à nouveau à l'horizon.

La routine meurtrière et l'horreur quasi quotidienne - massacres, faux barrages, femmes enceintes éventrées, bébés calcinés, cadavres sans têtes jetés au milieu de l'asphalte, policiers égorgés, jeunes militaires raflés, usines incendiées, paysans rackettés, jeunes filles kidnappées, villageois massacrés dans l'obscurité de la nuit, femmes violées et la liste des atrocités est longue - créent inéluctablement des replis psychologiques et des mécanismes psychiques de défense. Les journalistes vivant au jour le jour cette situation, en recevant des images cauchemardesques, des dépêches horrifiantes et des informations alarmantes, se soumettent au format de l'objectivité qu'impose leur métier. La fiction intervient alors, parfois longtemps après le retour à la paix comme dans ce roman, en tant que catharsis, d'autant que la politique de la "Réconciliation nationale », 
juridiquement la "Concorde civile »" a fait le choix d'effacer le passé, de refouler les mots, de cacher les cadavres... Ce passé est occulté et sans bilan. Il est demandé aux Algériens de pardonner et d'oublier ${ }^{7}$. «Ce livre est une sorte de thérapie qui vous permet de vous reconstruire, de mettre noir sur blanc un traumatisme dont peu de gens parlent»(15) précise l'auteur. Le passage de l'écriture journalistique à l'écriture romanesque constitue ici l'acte de s'allonger sur le divan du thérapeute, faire le jeu d'association de mots et remonter des fragments de l'inconscient en surface pour que les angoisses prennent corps. C'est ce qui donne une illusion du réel.

\section{L'illusion du réel}

On trouve d'ailleurs une certaine similitude entre des passages de ce roman et le reportage (Oussad) de l'auteur sur sa rencontre avec Ahmed Benaïcha, plus particulièrement la description de la route, de l'atmosphère, et du périple qui l'a amené jusqu'à son but. La structure de la quatrième partie, la plus longue, intitulée «La rencontre » (37-88), est quasi identique à son reportage journalistique, à la différence de la subjectivité intrinsèque à l'écriture romanesque. Le reportage se termine par la phrase : «Armé d'un Kalachnikov, il prend place à nos côtés. L'interview peut commencer », alors qu'à la fin de cette partie du roman, on peut lire : «Les présentations faites, l'interview pouvait commencer» (88). Le passage du factuel à la fiction s'exprime ici par le truchement du mode de la narration, le présent pour le reportage et l'imparfait pour le roman. Dans le reportage, l'écriture est «neutre». Mais dans la fiction, le personnage journaliste laisse libre cours à ses sentiments et pensées :

Je ne voulais pas lui répondre, me contentant de suivre les lignes blanches discontinues. La route avait ce quelque chose de magique qui me transportait loin de la réalité du quotidien. Les poteaux électriques étaient une haie d'honneur qui veillait sur moi. Les bornes kilométriques, des nains de jardin qui vous souhaitaient à chaque fois la bienvenue. Le bitume, un tapis rouge qu'on déroulait à ma rencontre. Les arbres, de vieux potes qui me reconnaissaient et

\footnotetext{
${ }^{6}$ «La Concorde civile » est une loi d'amnistie adoptée le 8 juillet 1999 par le parlement algérien. Un référendum la plébiscite avec $90 \%$ de votants selon les chiffres officiels le 16 septembre 1999. Cette loi avait pour but de réintégrer dans la vie civile les terroristes qui renonçaient à la violence, y compris les personnes impliquées dans les réseaux de soutien aux groupes armés. Une nouvelle loi, «La Charte pour la paix et la réconciliation nationale », modifiant la première, est adoptée toujours par référendum le 15 août 2005, permettant d'abandonner les poursuites judiciaires contre les terroristes qui déposaient les armes et qui n'étaient pas coupables de crimes de sang, de viols et d'attentats à l'explosif. ${ }^{7}$ Dans des pays ayant connu des conflits armés, à l'exemple de l'Afrique du Sud et de la Colombie, la réconciliation est passée par la libération de la parole où l'accusé en reconnaissant la totalité de ses actes demande pardon à la victime.
} 
déployaient leurs branches dans un au revoir figé pour l'éternité. La voiture, un vaisseau sans amiral qui voguait au gré de mes rêveries et les reportages, des missions que je devais mener à bien en compagnie de Kader, mon écuyer (56).

L'auteur joue avec l'intertexte en faisant référence à plusieurs personnages historiques de cette tragédie et à des événements sanglants, particulièrement des massacres de villageois, des déclarations d'hommes politiques, des analyses journalistiques et en évoquant des personnalités assassinées (le chanteur Hasni et le dramaturge Abdelkader Alloula). Mais le mot «terrorisme» est quasi absent et utilisé uniquement à deux reprises: une fois dans un extrait d'article inséré dans la fiction et la seconde fois dans un commentaire fait par le personnage Houari, alors que le terme «peur» revient une soixantaine de fois, montrant toute l'ampleur psychologique du passé.

Dans Les Chemins Inutiles, l'auteur joue des différents registres de langue et utilise les codes du roman noir, avec un personnage principal qui fume et boit de l'alcool régulièrement, qui est pessimiste, cynique, angoissé, et qui est à la fois apeuré et audacieux, sans oublier l'humour noir qui le caractérise. Bien que centré sur un personnage principal omniprésent et son intériorité, ce récit insère des coupures de journaux quelquefois postérieures à cette décennie -, introduit des «fiches signalétiques ${ }^{8}$ et même une légende connue dans la région de Aïn Temouchent sur le sort maléfique d'un virage de la route nationale : «On raconte que des automobilistes, sans aucune raison apparente, viennent s'écraser contre lui [un olivier solitaire] » (31). Il y a alors un jeu de miroir entre le factuel et l'imagination. C'est tout l'intérêt de l'intertexte et de la référentialité, par conséquent des extraits d'articles introduits, pour la plupart des éditoriaux - des repères extradiégétiques discursifs et temporels -, dans cette fiction. Le factuel qui est ici l'information, avec son corollaire le commentaire d'analyse, montre ce jeu de passage d'un mode narratif à un autre, plus particulièrement d'une forme d'écriture qui bannit le «je» au récit où domine la première personne du personnagenarrateur.

Le discours narratif de cette œuvre est marqué par des ruptures chronologiques manifestes. Les multiples analepses donnent l'illusion de revenir constamment au passé alors que l'intrigue avance. N'oublions pas que le rôle des réminiscences est de réintroduire le passé dans le présent.

8 Chaque fiche précise les nom, prénom, taille, poids, couleur des yeux, couleur des cheveux et caractéristiques particulières d'un personnage ou d'un objet (par exemple, la voiture du personnage Kader utilisée pour leur déplacement). 
Ce récit fonctionne en spirale d'analepses nettement plus récurrentes que les prolepses. Derrière chaque souvenir transparaissent la dangerosité des missions et la violence du terrorisme. Ce n'est qu'en toute fin de récit que le lecteur comprend que celui qui raconte, le personnage du journaliste, est un homme interné dans un hôpital psychiatrique. L'intrigue de ce roman se déroule en une seule et unique journée, celle où le journaliste a perdu son alter ego, son collègue photographe. Il se remémore plus de six ans plus tard sa mort et d'autres événements dispersés dans le temps. Les péripéties s'enchaînent entre le prologue, réintroduit tel quel vers la fin du récit, et l'épilogue où l'on saura que son collègue est venu le chercher pour aller faire un reportage. L'usage abusif des analepses, qui pourrait dérouter le lecteur, est balisé et atténué par la division du récit en cinq chapitres titrés, un prologue sans titre, mais un épilogue signalé, auquel il faut ajouter les "fiches signalétiques » et autres indices paratextuels, y compris la photo de la couverture choisie par l'auteur et qui n'est autre que la façade du marché Michelet au centre-ville d'Oran. Celle-ci montre des escaliers qui mènent vers un mur, une impasse. Les anachronismes balisés, le lecteur peut alors restituer les différents fragments du discours et la matrice principale du récit.

\section{Complicité d'un photographe myope et d'un narrateur « fou »}

Le personnage du journaliste a un compagnon omniprésent. Tous les deux se chamaillent continuellement. C'est leur seul mode d'échange. Kader est le photoreporter du journal. Pour une personne censée capter des images, figer un moment de la vie sur papier et surtout la tragédie dans le contexte de guerre, il est étrangement myope, «autant qu'une taupe atteinte de cataracte »(27). Cette métaphore n'est pas sans amplifier la difficulté à capter et traduire des situations de terreur. Il faudrait préciser que durant la décennie noire d'innombrables nouvelles n'ont pas été rapportées directement, mais communiquées par les différents corps de sécurité. Les journalistes les diffusaient, parfois au conditionnel, sans pouvoir aller sur place et vérifier ainsi les faits avant le bouclage du journal. Une simple consultation des archives peut fournir une idée plus claire et plus précise de la difficulté des journalistes à recouper l'information dans un contexte de conflit meurtrier. La terreur était quasi quotidienne alors que les journaux n'avaient ni la possibilité ni le temps de vérifier et de croiser leurs sources, notamment le soir, moment d'une 
totale insécurité; un couvre-feu ayant été décrété par l'État algérien'. En règle générale, l'information de "dernière minute » était divulguée en brève avant le bouclage et l'envoi du journal à l'imprimerie. Le lendemain et après des investigations, un article était rédigé avec plus de précisions.

Il faut bien situer cette pratique dans son contexte. Les terroristes n'avaient pas la possibilité, ou tout du moins très réduite, de produire et faire circuler leurs propres informations et images de propagande, comme c'est le cas aujourd'hui avec le développement d'internet et des outils et moyens de communication (téléphones portables, chaînes d'information en continu, réseaux sociaux). L'attaque des deux Tours jumelles du World Trade Center, le 11 septembre 2001, illustre par ailleurs cette information quasi instantanée retransmise en boucle dans le monde entier. La seconde révolution numérique du nouveau millénaire permet aujourd'hui aux terroristes de fabriquer leurs propres images, tout en utilisant les médias traditionnels.

Pour ce qui est de l'Algérie, les journaux avaient dans leur majorité fait le choix de ne pas diffuser les images les plus choquantes et les plus traumatisantes, pour ne pas faire le jeu de propagande des groupes armés et pour ne pas heurter la sensibilité des gens. Les rares rédactions qui ont décidé de ne rien dissimuler, dont le quotidien francophone Le Matin, ont suscité de vives polémiques à l'occasion de certaines photos arborées en Une. Les images choquantes diffusées, y compris à la télévision, sont surtout celles des terroristes abattus gisants au sol, souvent sales et à la barbe fournie, pour saper le moral des groupes armés et dissuader les potentiels candidats de les rejoindre. La représentation de la tragédie algérienne à l'étranger était dominée par l'allégorie au lieu d'image-choc, de sang et de sanglots, de cendres et de cadavres. Le cliché par exemple qui a fait le tour du monde, en septembre 1997, intitulé «La Madone de Bentalha», une femme en deuil pleurant la perte des membres de sa famille, suggère fortement dans l'imaginaire occidental l'iconographie chrétienne (Maazouzi) ; aucune tache de sang ne vient dénuder l'horreur.

\footnotetext{
9 Après un état de siège de quatre mois instauré en juin 1991 pour faire face à l'insurrection islamiste, l'état d'urgence est décrété le 29 février 1992. Un couvre-feu partiel, de minuit à quatre heures du matin, est instauré également en décembre 1992 pour 7 wilayas, puis 10 wilayas (départements). Tandis que le couvre-feu prend définitivement fin en février 1996, l'état d'urgence est levé le 24 février 2011, dix-neuf ans après son instauration. Les personnes évitaient de circuler ou de sortir en fin de journée dans des régions rurales et dans des villes, particulièrement la capitale Alger.
} 
Kader le myope dans Les Chemins inutiles dénote une forme d'autocensure pratiquée par le journalisme algérien par choix (déontologique ou autre), notamment en ce qui concerne les images. La myopie symbolise la fragilité et la délicatesse, voire l'impossibilité, de rapporter des moments de terreur et de drames. Le personnage principal et son adjuvant Kader symbolisent ce qui est absent ou indicible dans l'article et l'image du journal. Photographier est forcément cadrer, s'arrêter sur le bout d'une réalité plus vaste, figer un mouvement, un instant, au moment de cliquer sur le déclencheur. La myopie peut avoir une autre symbolique : ce n'est en effet pas juste mal voir, c'est ne voir bien que de près; le reste est flou. Kader, qui a une longue expérience en tant qu'ancien employé de l'unique agence officielle du pays et qui a longtemps voyagé à l'étranger dans le cadre de couverture des visites de présidents algériens, est constamment sur le terrain, sur les lieux des drames, au plus près des victimes du sang pour les voir ${ }^{10}$.

Dans ce roman, le drame refoulé par le journaliste est pris en charge par le romancier. C'est pour cela que Les Chemins inutiles ne constitue pas un roman de témoignage. D'ailleurs, le doute s'installe à la fin du roman sur la crédibilité de l'instance narrative. On découvre que le personnagenarrateur raconte son récit depuis un asile où il est interné. Or il n'est pas un patient comme les autres puisqu'il est bien conscient de l'endroit où il se trouve, puis décrit et se moque de la manie de chacun des pensionnaires à l'hôpital psychiatrique d'Oran. La salle 2 qu'il occupe «était celle des fous mécanisés, des maboules dans la peau d'avions, de locomotives ou encore de voitures, pour les moins prétentieux, ce qui était occasionnellement mon cas. La salle 3, à quinze mètres de nos grillages, était le siège des fous politisés » (137).

Dans une autre séquence moins explicite au début du roman, le personnage principal semble avoir entendu la voix d'une femme, une sorte de spectre qui lui a rendu visite dans son studio du quartier mal famé de Saint-Pierre (les toponymes dans ce roman sont factuels) et le suit partout dans ses hallucinations. Cette folie du personnage-narrateur, plus explicite et inattendue à la fin du roman, invite à la relecture du récit et à une nouvelle interprétation. Ce procédé fait ainsi planer un doute sur la parole $\mathrm{du}$ journaliste et sur la véracité des événements. Cette folie exprime la difficulté à rationaliser la violence dans sa totalité. Ce roman explore les ravages du terrorisme sur les individus et le drame algérien sans un véritable bilan, à travers la «schizophrénie» du personnage principal,

${ }_{10}$ Précisons que les journaux algériens n'avaient pas les moyens d'utiliser les couleurs et les photos étaient en noir et blanc. Le conflit était donc illustré en clair-obscur. 
plutôt son état d'entre-deux, entre rationalité et folie. Les deux journalistes sont à la fois lucides et paranoïaques, atteints d'hallucination. Kader pense régulièrement déceler une "présence ». Une nuit, il voit en la lumière aveuglante d'un hélicoptère de l'armée qui les a survolés, « un ange » (114). Le délire et la figure du fou revêtent une dimension réfractaire dans la littérature algérienne. Le fou participe à la transgression des conventions sociales et politiques. Dans la société, il a une double image; accepté et craint. Ses paroles sont écoutées et révélatrices de vérités inavouées.

Cette complicité entre un myope et un « fou » renvoie par ailleurs à la précarité $^{11}$ d'un métier en contexte de guerre, au courage d'aller à la rencontre des drames, mais aussi à la difficulté de transmettre, de rendre lisibles, voire tangibles ces situations; certains crimes étaient inimaginables. Les deux journalistes communiquent de manière légère et persiflent afin d'éviter de parler de choses sérieuses qui peuvent être funestes pour leur moral. Il leur arrive aussi d'exprimer leurs souffrances en pleurs ou en se bagarrant.

\section{Dualité journaliste/terroriste et meurtre symbolique}

Dans de nombreux romans, la figure du terroriste est liée à une problématique sexuelle (ce que fait l'auteur en partie avec le personnage de Mustapha Abou Qatada) ou à un cas pathologique, quelle que soit l'aire culturelle ou l'époque, en occultant la dimension politique (Granier). L'intérêt du roman Les Chemins inutiles est de mettre l'accent sur la dimension idéologique, les actes des terroristes - ce qu'ils ont fait subir aux populations - et la guerre fratricide qui a déchiré leurs rangs. Puis, tout au long de l'intrigue, Mustapha Abou Qodata, émir qui a refusé la reddition, pourchasse le journaliste partout pour l'éliminer.

Cette obsession et cet acharnement, le journaliste qui a rencontré, défié et reçu une correction de Mustapha au maquis, ne les comprend pas et ne les explique pas. Il n'aura jamais de réponse à ses questions, puisqu'au hasard de la route, lors du retour d'une couverture dans un village attaqué près de Tlemcen, l'émir Mustapha, toujours à l'affût d'une embuscade contre le journaliste, est renversé dans le brouillard par la voiture de Kader. Pensant d'abord que le véhicule a percuté un animal et

${ }^{11}$ Malgré leur courage et leur engagement, les journalistes algériens travaillaient pour la majorité dans une totale précarité, sans statut et sans couverture sociale, alors que de nombreux éditeurs se sont enrichis. Le roman Les Chemins inutiles évoque cette précarité : Kader travaille au noir et le journaliste-narrateur a peur d'être licencié par son rédacteur en chef qu'il n'apprécie pas. 
qu'il faut fuir les lieux, le journaliste s'entête à vérifier l'origine du choc. Avant de rendre son dernier souffle, l'émir tire une balle sur son adversaire et le rate. Le journaliste s'acharne alors sur le cadavre à coups de pieds comme pris de démence et laisse éclater sa rage.

Contrairement au reportage et à Benaïcha, acteur de négociation de la paix, Mustapha Abou Qotada est une pure invention du romancier. Abou Qodata est l'archétype du terroriste sanguinaire, à la personnalité complexe, qui refuse la défaite :

Sa démarche féline contrastait singulièrement avec sa carrure de lutteur de foire $[\ldots]$ Il avait le cou démesurément long et pratiquement interminable lorsqu'il inclina la tête vers l'arrière [...] Mustapha ferma les yeux et, le temps d'un battement de cils, ses traits tracés au burin retrouvèrent la sérénité d'un petit enfant insouciant [...] Il (le journaliste) n'avait jamais rencontré un regard d'une telle intensité bestiale. Glacial. Des yeux de prédateur, véritable fenêtre donnant tout droit sur l'enfer. (4647)

Cette dualité journaliste/terroriste offre un aperçu de la relation délicate de dépendance entre ces deux protagonistes, l'un cherchant l'information pour ses lecteurs et l'autre la publicité pour ses actions, parfois dans une quête commune de notoriété. Il arrive que cette accointance se termine dans le drame, à l'image des journalistes décapités ou kidnappés en échange de rançons. La rencontre avec un terroriste est fantasmée dans les salles de rédaction comme est rêvée chez le thérapeute celle de la victime et son bourreau. Une sorte de fascination, qui pourrait être morbide, malsaine ou voyeuse, les unit. Le terroriste, qui fait et commande l'événement, créé et produit du travail pour le reporter, même dans le cas particulier où les journalistes sont directement ses cibles. Le face-à-face est alors explosif, une sorte de duel à mort entre les deux acteurs. Le terrorisme contemporain joue d'ailleurs de cette interdépendance en rendant ses actions plus retentissantes pourvu qu'elles restent des jours ou des semaines dans l'actualité.

Dans cette œuvre, le fait que la victime tue son bourreau par inadvertance et s'acharne ensuite sur ce cadavre montre l'impuissance du reporter à maîtriser la situation et à protéger sa vie. Cette séquence du roman est aussi l'image de la victoire du journalisme sur le terrorisme pour une presse algérienne très engagée. Contrairement aux journalistes étrangers à l'affût de scoop et de la rencontre des membres des groupes fondamentalistes armés qu'ils qualifiaient de rebelles, de guérilleros, 
d'opposants $^{12}$, la parole des terroristes était quasi absente dans la majorité de la presse algérienne. Saïd Oussad a d'ailleurs subi des critiques virulentes de ses confrères lorsqu'il a interviewé Ahmed Benaïcha, alors que le pays sortait de l'intensité de la guerre contre les civils lancée par les groupes armés et de sa phase la plus meurtrière (1993-1998).

Il faut dire que la corporation journalistique en Algérie a payé un lourd tribut : une centaine de journalistes ont été assassinés et d'autres ont choisi l'exil (Labter). La figure emblématique et mémorielle de ce sacrifice est le journaliste, poète et écrivain Tahar Djaout, abattu en plein jour devant son domicile. Le choix de l'auteur de ne pas donner de patronyme au personnage-narrateur est une façon de rendre hommage à tous les journalistes. On peut le lire aussi comme une reprise de l'anonymat qui caractérisait la pratique journalistique en Algérie, car de nombreuses personnes signaient leur article avec un pseudonyme par précaution et dans la crainte d'être repérées. Un autre phénomène marquant de cette décennie est la venue à la littérature de nombreux journalistes; ils constituaient presque la moitié des auteurs. C'est dire combien les journalistes étaient en prise directe avec la situation sécuritaire et le contexte, même si la venue à la littérature de nombre d'auteurs s'insérait dans une stratégie de placement dans le champ littéraire, plus particulièrement en France (Leperlier), en utilisant le capital symbolique de la corporation.

Les Chemins inutiles de Saïd Oussad, même s'il reprend de nombreuses caractéristiques $^{13}$ de la littérature algérienne de cette décennie marquée par un "palimpseste du sang » (Boujedra), revient surtout sur le trauma refoulé et questionne les mécanismes psychologiques de défense des individus en contexte de terreur. "J'avais mon indifférence et lui son cynisme pour nous protéger » (92) dira le personnage-narrateur de lui-même et de son collègue photographe, alors qu'ils assistent dans un cimetière à l'enterrement de vingt villageois "passés à l'arme blanche » (91). L'auteur ${ }^{14}$ s'est inspiré de son expérience de journaliste au plus près de l'événement pour explorer les états indicibles de l'individu dans le feu de l'action, tamiser les traumatismes d'une époque qui reste à examiner en

12 Des journaux étrangers doutaient des actes des terroristes en les imputant à l'armée algérienne, ce que résumait le fameux questionnement "Qui tue qui? ». À propos de la presse française (Hadj Ali)

${ }^{13}$ Hadj Meliani signale la masse de productions - se chiffrant à un millier d'ouvrages - en France sur l'Algérie à cette époque. Ces œuvres de fiction prenaient souvent deux formes : l'autobiographie réelle ou feinte et le roman à thèse (Meliani)

14 Une réédition remaniée du roman Les Chemins inutiles devrait paraitre prochainement aux éditions Frantz Fanon en Algérie. 
profondeur. Les stigmates de cette période sont encore présents sur les façades des habitations, à l'image des fenêtres grillagées, des doubles portes blindées ou des barrages permanents de militaires à l'entrée des grandes villes ou bien dans l'attitude de méfiance des individus. Il est connu qu'après des phases d'amnésie et de refoulements, les phénomènes de violence extrême ressurgissent pour interroger les sociétés, l'homme sur ses limites et ses radicalités, son humanité et sa barbarie, en rappelant les sacrifices et en réveillant les spectres du passé. Ce roman de Saïd Oussad sonde les blessures «béantes»(Djahnine) du conflit armé. Les Chemins inutiles sont la parabole des voies funestes et du temps perdu pour enfin aboutir à la paix après un bilan de milliers de victimes et de morts. 


\section{Bibliographie}

Djahnine, Habiba. «Le corps de l'Algérie porte une blessure béante. Il est temps qu'on la voie » (entretien réalisé par Adli Meddi), in El Watan, $1^{\text {er }}$ juillet 2011. Web. Consulté le 17 juin 2017.

Granier, Caroline. «La représentation du terroriste anarchiste dans quelques romans français de la fin du XIXe siècle», in Cabier d'Histoire: Revue d'bistoire critique, n 96-97, 2005, pp. 137-157. Web. Consulté le 17 juin 2017.

Hadj Ali, Youcef. Lettre ouverte aux Français qui ne comprennent décidément rien à l'Algérie. Albin Michel. 1998.

Labter, Lazhari. Journalistes algériens entre le bâillon et les balles. L'Harmattan, 1995.

Leperlier, Tristan. "Journaliste dans la guerre civile algérienne: Une profession intellectuelle entre littérature et politique », in L'Année du Maghreb, n 15, Paris, 2016. Web. Consulté le 17 juin 2017.

---. Une guerre des langues? Le champ littéraire algérien pendant la " décennie noire » (1988-2003): Crise politique et consécrations transnationales. Thèse de doctorat, École des hautes études en sciences sociales (EHESS), 2015.

Maazouzi, Djemaa. "Arrêt sur un massacre, image d'une guerre ", in Hors champ, Montréal 15 octobre 2006. Web. Consulté le 17 juin 2017.

Meliani, Hadj. Une littérature en sursis? Le champ littéraire de langue française en Algérie. L'Harmattan, Paris, 2002.

Mokthari, Rachid. La Graphie de l'horreur. Chihab, Alger, 2003.

Oussad, Saïd. Les Chemins Inutiles. L'Harmattan, 2014.

---. «Je suis un écrivain d'Instinct» (Entretien avec Mohammed Yefsah), in Les Débat, $\mathrm{n}^{\circ}$ 1170, jeudi 11 décembre 2014, p. 15.

---. "Chlef: À la rencontre de l'AIS », in La Voix de l'Oranie, lundi 17 janvier 2000. 